\title{
The Role of the Psychiatrist in the Pain Clinic
}

\author{
StePhen P. TYrer, Senior Lecturer in Psychiatry, Research Unit in Psychological Medicine. University of Newcastle upon
}

Tyne

In Britain there are almost 200 pain clinics concerned with the diagnosis and treatment of patients with chronic intractable pain. These patients have frequently run the gamut of medical and surgical services and they are referred to a pain clinic for further assessment and treatment, especially those cases where no organic basis can be found to account for the complaint of pain. Other reasons for referral include specialist treatment only available in a pain clinic, where conventional therapy has failed or where further surgical or medical treatment is not considered justifiable.

A wide variety of painful conditions are seen in the pain clinic. The most common presenting complaints are backache, pain from nerve damage, e.g. post-herpetic neuralgia or trauma, headache and pain from malignant disease. Unsurprisingly, many patients with chronic pain are dissatisfied and unhappy, and these include a substantial proportion who are found to have psychiatric illnesses. The percentage of patients with psychiatric diagnoses ranges from 10 per cent to 90 per cent depending upon how the evaluation of mental illness is made. Recent surveys using established psychiatric schedules have shown that $30-50$ per cent of patients. have measurable psychiatric disturbances. Two-thirds of these patients have a major depressive disorder as diagnosed by DSM-III criteria. The remainder have personality disorders. anxiety states, hysteria and drug dependence with the occasional patient with paraphrenia or organic brain syndrome.

Virtually all British pain clinics are directed by anaesthetists. A minority of clinics are run in collaboration with specialists from other disciplines which may include physicians, surgeons, pharmacologists, psychiatrists and psychologists. The majority of pain clinics are out-patient facilities. and units with in-patient beds and a regular multidisciplinary input are relatively rare. Although many of the personnel working in these clinics have wide experience of pain states. their knowledge of psychiatric illness is understandably limited, and it has been shown that psychiatric diagnoses are missed. This will be a particular problem in those clinics without ready access to psychiatric or psychological help.

To make matters more difficult for the non-specialist, many patients attending pain clinics have neither circumscribed organic nor psychiatric conditions. In some of these patients the exhibition of pain behaviour has enabled the patient to avoid unpleasant situations or has been followed by pleasurable consequences, e.g. sympathy from a relative or nurse, opportunities to converse about symptoms, the ability to avoid unpleasant situations or financial gain. Because this behaviour is followed by a rewarding result it tends to become more frequent and has been described as learned pain behaviour or abnormal illness behaviour. These patients are often skilled manipulators who are unable, or unwilling, to give up the sickness role. They require identification and treatment, usually by behavioural means.
Pain clinic physicians are not formally trained in the identification and management of these patients and thus there are excellent grounds for suggesting that psychiatrists and psychologists should be regular members of pain clinic teams. They are in a better position to identify patients with psychiatric and psychological problems and have greater expertise and skill in selecting and carrying out appropriate treatment. The problem facing those pain clinic physicians without ready access to psychiatric or psychological help is how to identify those patients whose condition requires these skills. A number of psychiatric and psychological self-rating instruments have been found to be valuable in detecting nonorganic illness in pain patients. Questionnaires that have been used to detect psychiatric illness include the Beck Depression Inventory, the Zung Self-Rating Depression Scale. The General Health Questionnaire and the Leeds Scales for Assessment of Depression and Anxiety. The Beck and Leeds Scales have been found to be most sensitive in this regard, although specificity is not so good.

The identification of the patient with learned pain behaviour is not so easy. The Minnesota Multiphasic Personality Inventory (MMPI) has been widely used in the assessment of patients with many different painful conditions. The so-called 'conversion V', with abnormal scores on the hysteria, hypochondriasis and depression scales of the MMPI, has been found frequently in patients with learned pain behaviour. However, recent work has suggested that these MMPI abnormalities are of limited value in predicting response to treatment. They may simply represent the personality attitudes of anybody with chronic intractable pain.' Similarly, the Illness Behaviour Questionnaire, designed to quantify learned pain behaviour by Pilowsky and Spence, ${ }^{2}$ reliably distinguishes patients with chronic pain from those attending general practitioners with acute complaints, many of which are trivial. However, within a chronic pain population this instrument was unable to discriminate between patients with organic, psychiatric and abnormal illness behaviour characteristics. ${ }^{3}$ It is apparent that it is difficult to identify these patients by means of self-report questionnaires, particularly as they are frequently inaccurate in describing their symptoms. These patients can only be detected reliably at interview.

The results of these studies suggest that a reasonable course of action would be to administer the psychiatric questionnaires to all patients attending the pain clinic and to refer all patients with high scores to a psychiatrist or psychologist. Any patient who displays pain behaviour, or who exaggerates his or her symptoms to a marked degree should also be referred.

Should these patients be seen by the psychiatrist or psychologist? The psychiatrist is able to use his knowledge of psychopharmacology to select drugs that may be suitable. A few patients have well-defined psychiatric syndromes that can be 
treated by conventional treatments, e.g. antidepressants for endogenous depression, neuroleptics for schizophrenia and monoamine oxidase inhibitors for phobic anxiety. In addition, many patients with persistent pain who are not suffering from depression may benefit from tricyclic antidepressant treatment in doses that are usually considered to be subtherapeutic in the treatment of depressive illness. The psychiatrist should also be able to identify features in the history of these patients that may provide the basis for future management. These include adoption of an adult role early in life with insufficient time or opportunity to be dependent on parents, evidence of rejection or physical abuse in childhood, history of excessive activity or hard work antedating the pain by many years, and existence of a crippled relative. With this background, social and marital difficulties are not uncommon.

Although the interpretation of the problems may not be too difficult, subsequent management is more likely to involve behavioural or cognitive methods of treatment, rather than psychotherapy. It has been realized for some years that many patients with chronic psychogenic pain are unable to understand or accept alternative explanations for the origin of their pain which fail to include physical factors. These patients often cannot verbalize their feelings and may be incapable of linking emotions with fantasies-a condition that has been described by the term alexithymia. ${ }^{4}$ It is understandable that patients of this kind are unlikely to be devotees of the psychotherapist's couch.

It is concluded that the psychiatrist plays a more limited role in the pain clinic than might be expected considering the frequency of psychiatric conditions in this population. He can be best used in identifying those patients with definitive psychiatric illnesses, treating those that are likely to benefit from drugs, and referring the remainder to behaviourally or $\operatorname{cog}$ - nitively-orientated psychologists. Psychiatric assessment scales for depression can be used as screening instruments and would avoid the psychiatrist having to see every patient attending the clinic. Even this limited service may be unnecessary. A recent survey from the Mayo Clinics showed that although 53 per cent of patients admitted to a pain centre were depressed on admission, only 2 per cent were depressed at discharge, despite receiving no specific pharmacological or other treatment for depression. Ipso facto, unless our psychiatrist is an experienced behavioural or cognitive therapist his skills may be uncalled for in a pain clinic setting.

\section{ACKNOWLEDGEMENTS}

My thanks are due to Dr J. E. Charlton and Professor J. W. Thompson of the Newcastle Pain Relief Clinic for vigorous criticism and helpful comments on the manuscript.

\section{REFERENCES}

iHagedorn, S. D., Maruta, T. Swanson, D. W \& Collugan, R. C. (1984) Premorbid MMPI profiles of low back pain patients: surgical successes versus surgical failures. Pain, Supplement 2, 258.

2Pliowsky, I. \& SPENCE, N. D. (1975) Patterns of illness behaviour in patients with intractable pain. Journal of Psychosomatic Research, 19, 279-287.

JTyrer, S. P., Capon, M. N., Peterson, D. M., Charlton, J. E. \& Thompson. J. W. (1985) The detection of psychiatric illness and psychological handicaps in a British pain clinic population. (Accepted for publication in Pain.)

4Sifneos, P. E. (1973) The prevalence of 'alexithymic' characteristics in psychosomatic patients. Psychotherapy and Psychosomatics, 22, 255-262.

SBrown, M. K., Maruta. T. \& Mchardy, M. J. (1984) Effectiveness of management of chronic pain in the long-term resolution of pain-associated depression. Pain, Supplement 2, 185.

\title{
Policy into Practice in a New Secure Unit
}

\author{
M. Faulx, Department of Psychiatry, Knowle Hospital, Fareham, Hants.
}

Albert Kushlick first showed me this technique. He used it for setting up hostels for the mentally handicapped and I then used it when setting up a Medium Secure Unit. It could be applied to any Unit and I am writing it up because I found that staff members in new units had run into problems where they had not adopted this or a similar approach. The idea is to show how one can translate 'aims' or 'principles' into 'brass tack' practices by design and with consultation in a multidisciplinary setting.

I make the assumption that the consultant in charge (who will be looked to to provide a lead) will, through study and experience, have gained a clear idea of the sort of unit and the sort of attitudes and practices he wishes to see developed. This is probably best obtained by visiting many units and taking from them their best ideas.

The next problem is how to convey these ideas to one's own staff and involve them in the process of finding the best way to do things. In my case I spent considerable time in seminars during a two-month induction period with the newly appointed staff of the Unit, in conveying the principles on which I wished to see the Unit run. Incidentally, I found that I learned a great deal from the staff, which expanded my own ideas. One has then to ask how can these principles be enacted or, more basically, who would do what and when, and who would take and pass on responsibility?

To decide this, the newly appointed staff and myself sat down together through a series of 'policy and practice' meetings. A leader (in our case, myself) acted as a conductor at the policy meetings. The first thing to do is to break the task down to a whole series of 'problems' which can be specified. Thus the task of 'How to create a pleasant environment' is broken down into: 'How do we keep the ward clean?'; 'How do we keep a constant supply of fresh linen?': 'How do we greet and admit a new patient?' etc. Thus these 'problems' vary from 